\title{
Estimating Human Interactions with Electrical Appliances for Activity-based Energy Savings Recommendations
}

\section{Motivation}

\section{Household Focus}

- Smart Home:

- Spread of communicating smart devices in the home

- Future availability of appliance-level consumption data

- Household-level optimization for load shifting, peak shaving:

- Accounting for the residents' lifestyle characteristics

- Ambient Intelligence System:

- Combination of real-time grid status/prices, in-home states, etc.

- Local (in-home) optimization $\rightarrow$ no privacy concerns

\section{Activity-based Recommendations}

- About $12 \%$ energy savings if personalized real-time feedback is employed [Carrie Armel et. all, Energy Policy, 2013]

- Relationship between human activities and succession of powered-on appliances

- Higher abstraction level for residents: activity vs. appliance-level feedback
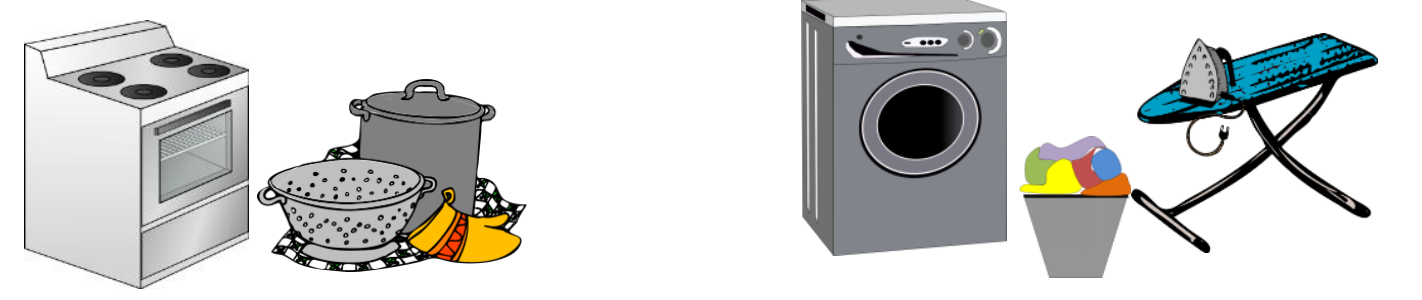

\section{Methodology}

\section{Appliances States}

- User interaction incurs changes from idle state (stand-by/off) to active state (powered on/in use)

- Single appliance-level data:

- Different operation modes (e.g. washing machine: soaking, spinning, etc.)

- Circuit-level data:

- Power strip, room-level, etc.

- Challenges:

- Different appliance types/models $\rightarrow$ different thresholds for idle/active

- Unsupervised threshold detection

\section{GMM}

- Power measurements distribution shifted towards lower values

- Readings resampling:

$$
C * \log \left(n_{i}+1\right)
$$

- States: differently shaped peaks in the distribution

- Best configuration (no.

Gaussians): lowest Bayesian Information Criterion (BIC) value

- Idle/active threshold: the first valley in the distribution

\section{Experiment}

- PecanStreet dataset

- 239 households with 1-minute power measurements

- January - May 2014

- 73 categories of readings collected

- At most 22 appliances/circuits per households

- No ground truth

- Ignore:

- Always-on devices (fridges)

- Devices consuming less than 0.5 Wh per week

\section{Results}

- Single appliance-level data (clotheswasher):

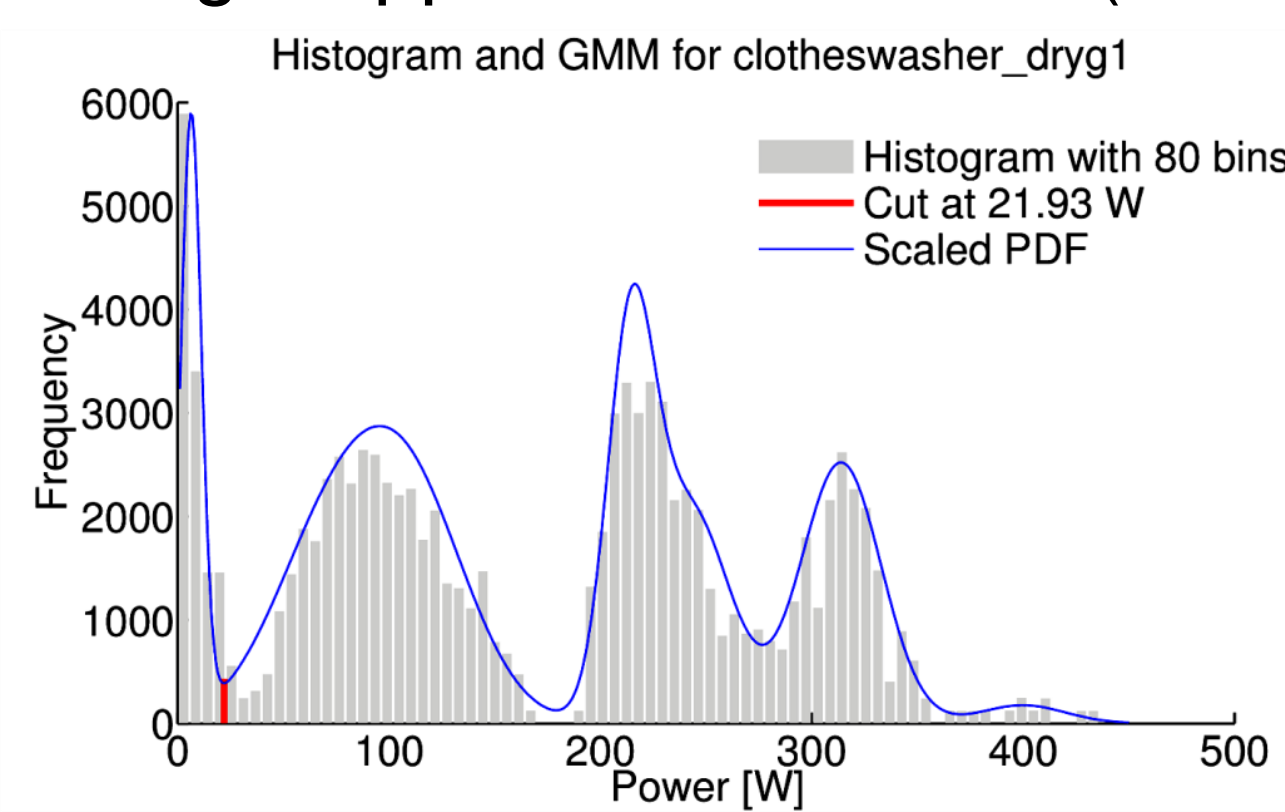

- Circuit-level data (kitchen):

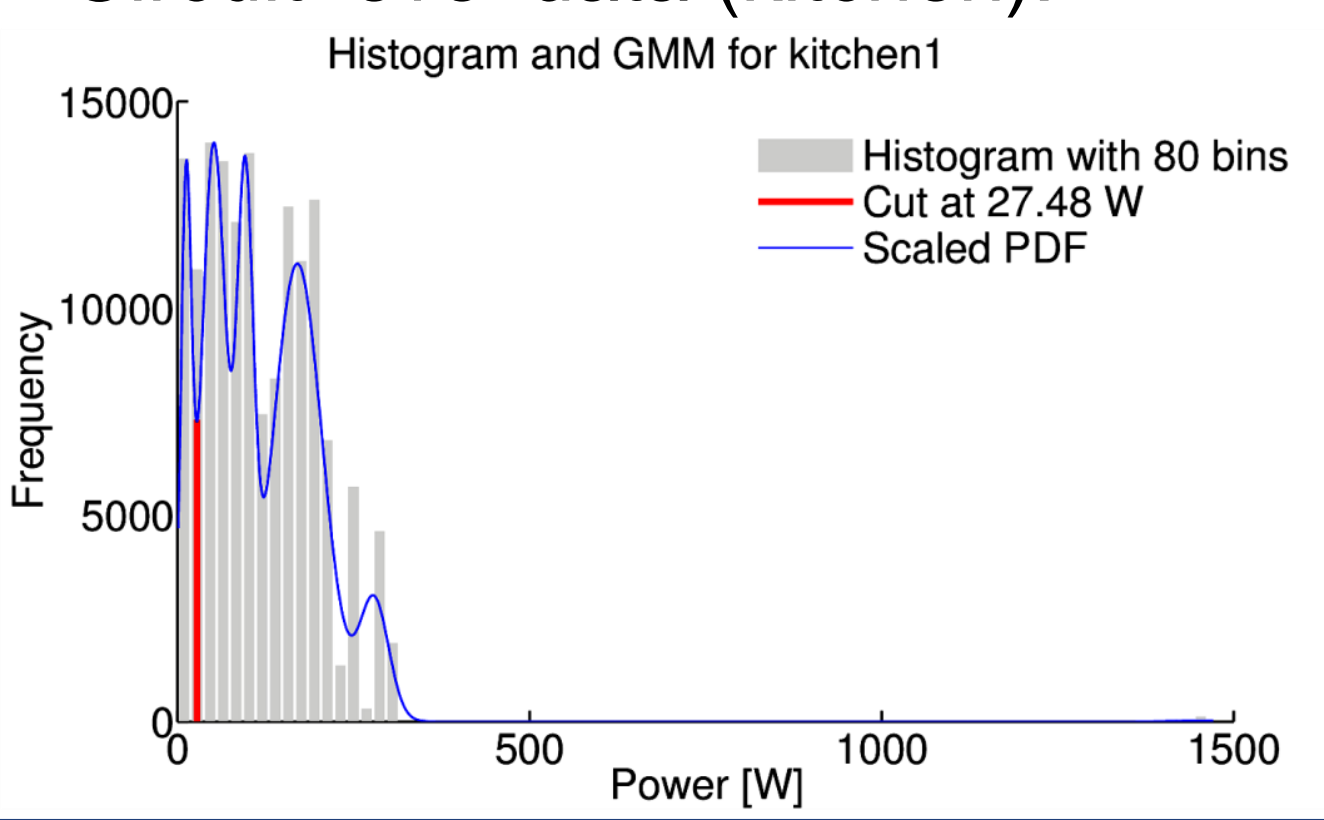

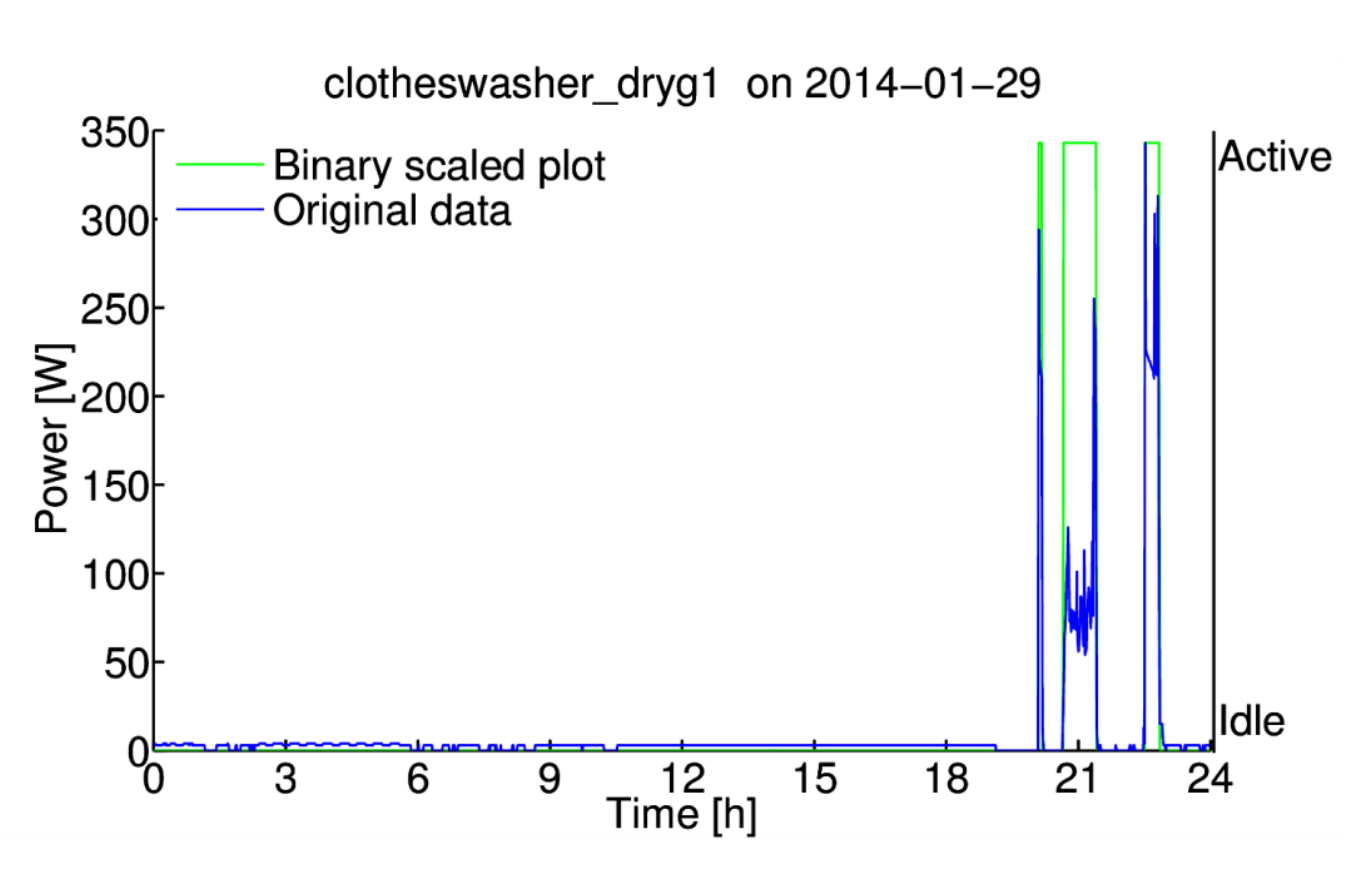

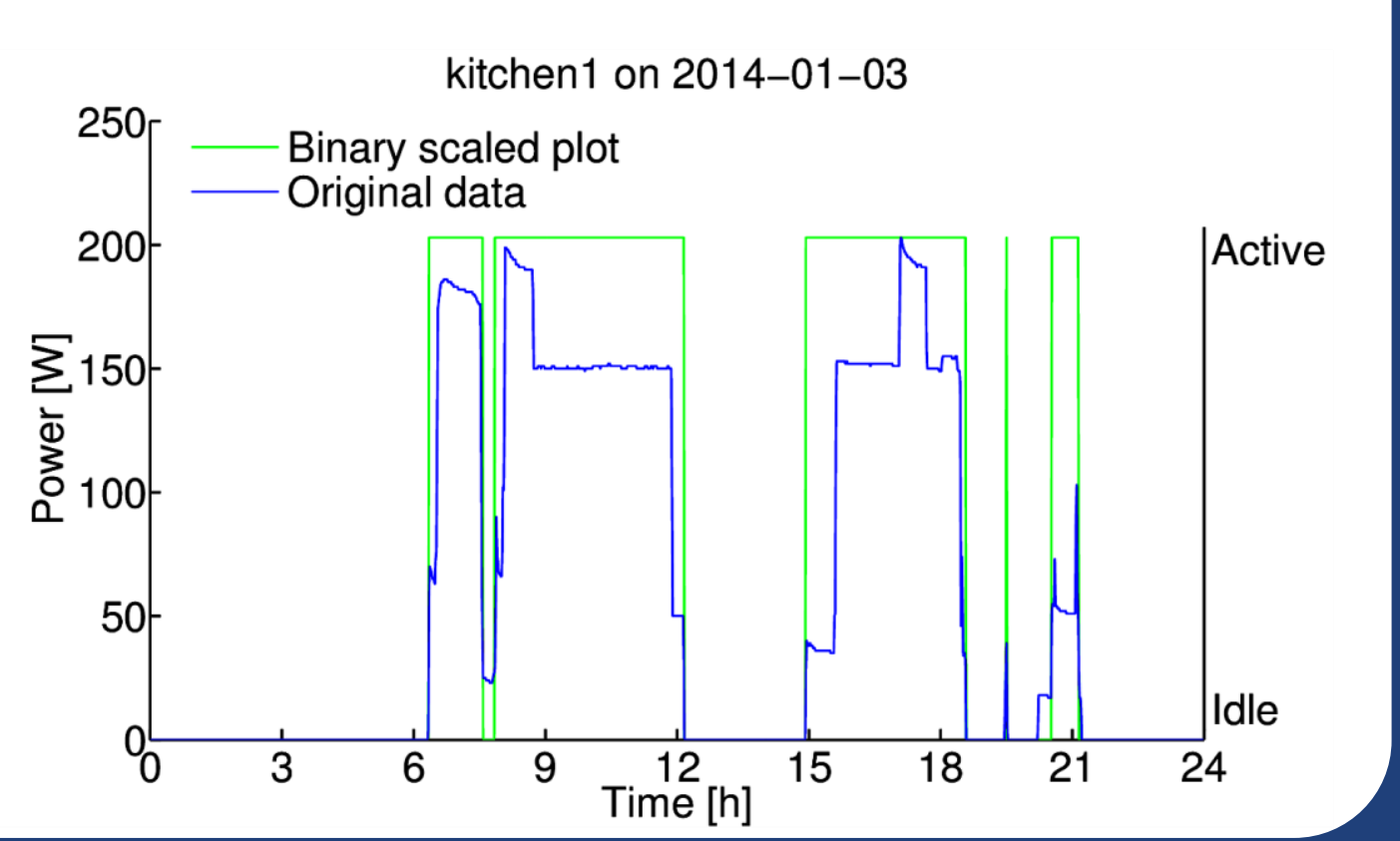

\section{Outlook}

- Summary:

- Modeling of appliance functioning through states

- States detections via re-sampled power readings distribution modeling with GMM

- Distinction between idle/active states based on the first valley between the first two Gaussians

- Future work:

- Thresholding Evaluation

- Ground truth acquisition

- Activities detection:

- Recurrent patterns of appliances usage

- Temporal rules 